\title{
Direct brain involvement of Takayasu arteritis treated with rituximab and infliximab: a case report
}

\author{
Seondeuk Kim ${ }^{1,2, *}$, Seon-Jae Ahn ${ }^{1,2, \star}$, Han Sang Lee ${ }^{1,2}$, Woo-Jin Lee ${ }^{1,2}$, Jangsup Moon ${ }^{1,2,3}$, Jin Kyun Park \\ Kon $\mathrm{Chu}^{1,2, *}$ \\ ${ }^{1}$ Department of Neurology, Seoul National University Hospital, Seoul, Korea \\ ${ }^{2}$ Laboratory for Neurotherapeutics, Center for Medical Innovation, Biomedical Research Institute, Seoul National University Hospital, \\ Seoul National University College of Medicine, Seoul, Korea \\ ${ }^{3}$ Rare Disease Center, Seoul National University Hospital, Seoul, Korea \\ ${ }^{4}$ Division of Rheumatology, Department of Internal Medicine, Seoul National University College of Medicine, Seoul, Korea
}

\begin{abstract}
Takayasu arteritis (TAK) is a systemic vasculitis involving large arteries. Reports of direct central nervous system (CNS) involvement in TAK are extremely rare in the literature. In addition, treatment for direct involvement has not been reported. Herein, we describe a case of encephalitis in a TAK patient who presented with fever and headache at the first attack, then cognitive impairment at the second attack. The patient improved with rituximab and especially infliximab. These findings indicate the usefulness of rituximab and infliximab to treat the direct CNS manifestations in TAK.
\end{abstract}

Keywords: Takayasu arteritis, Central nervous system, Encephalitis

\section{Introduction}

Takayasu arteritis (TAK) is a systemic vasculitis involving large arteries, such as the aorta and its main branches (e.g., subclavian, brachiocephalic, extracranial arteries) [1].

The central nervous system (CNS) manifestations (e.g., ischemic stroke) in TAK are usually associated with occlusion or stenosis of large arteries [2,3]. However, direct CNS involvement in TAK (e.g., intracranial granulomatosis) is extremely rare $[2,4]$. Furthermore, reports regarding the treatment of CNS involvement were not found in a literature review.

Glucocorticoids are the treatment of choice for TAK [5]. However, approximately two-thirds of patients experience disease recurrence or steroid dependency. Therefore, other immune suppressants are often used as additive or alternative treatments for corticosteroids. The effectiveness of rituximab as second-line treatment for TAK was shown in several studies [6]. Infliximab, a tumor necrosis factor (TNF) inhibitor, was also used to treat refractory TAK with good efficacy [7]. Herein, we describe a case of autoimmune encephalitis in a patient with TAK who showed good clinical response to rituximab and radiological response to infliximab.

\section{Case Report}

A 39-year-old male presented with severe headache, myalgia, and mild fever for 2 months. He was admitted for evaluation of prolonged fever. The patient did not complain of other symptoms (e.g., cough, sputum, rhinorrhea, diarrhea, arthral-

Received: July 23, 2021 Revised: August 27, 2021 Accepted: August 27, 2021

Correspondence: Kon Chu

Department of Neurology, Seoul National University Hospital, 101 Daehak-ro, Jongno-gu, Seoul 03080, Korea

E-mail: stemcell.snu@gmail.com

ORCID: https://orcid.org/0000-0001-5863-0302

*These authors contributed equally to this study as co-first authors. 
gia, claudication, dizziness, dysarthria, or sided weakness). His blood pressure was mildly elevated (systolic blood pressure 140-150 $\mathrm{mmHg}$ ) without a significant difference ( $>10$ $\mathrm{mmHg}$ ) between both arms, and his body temperature was above $38.0^{\circ} \mathrm{C}$. Upon physical examination, no oral/genital ulcers or skin rashes were observed. In addition, radial and dorsalis pedis artery pulses were present. Furthermore, bruit was not observed in either the carotid or ophthalmic arteries. The detailed neurological examination revealed no focal neurological deficits, including spasticity or dyskinesia. Computer tomography (CT) of the abdomen and pelvis was unremarkable; however, chest CT revealed wall thickening of the ascending aorta, right proximal brachiocephalic artery, and left proximal common carotid artery (Figure 1A). Brain magnetic resonance imaging (MRI) showed T2 high signal intensities and $\mathrm{T} 1$ contrast-enhanced lesions involving the right basal ganglia, corpus callosum genu, and both hypothalamic areas without stenotic or occlusive lesions in the intracranial and extracranial arteries (Figure 1B-E). CNS vasculitis, demyelinating disease, brain tumor, autoimmune encephalitis, and infectious encephalitis were included as differential diagnoses. Torso 18F-fluorodeoxyglucose (FDG) positron emission tomography (PET) showed no evi-dence of tumorous conditions. In particular, brain FDG-PET showed hypometabolism of the affected lesions (Figure 1F). Based on a cerebrospinal fluid analysis showing a normal profile, other infectious etiologies were also excluded. The autoantibodies and the vasculitis-associated tests were unremarkable. Unspecified inflammatory CNS diseases such as CNS vasculitis or demyelinating disease were suspected, and an intravenous (IV) corticosteroid pulse was administered. After pulse therapy, radiologic findings and the symptoms (fever and headache) improved. The oral steroid was maintained and then tapered off over an approximately 1-month period.

Figure 1 Initial imaging findings
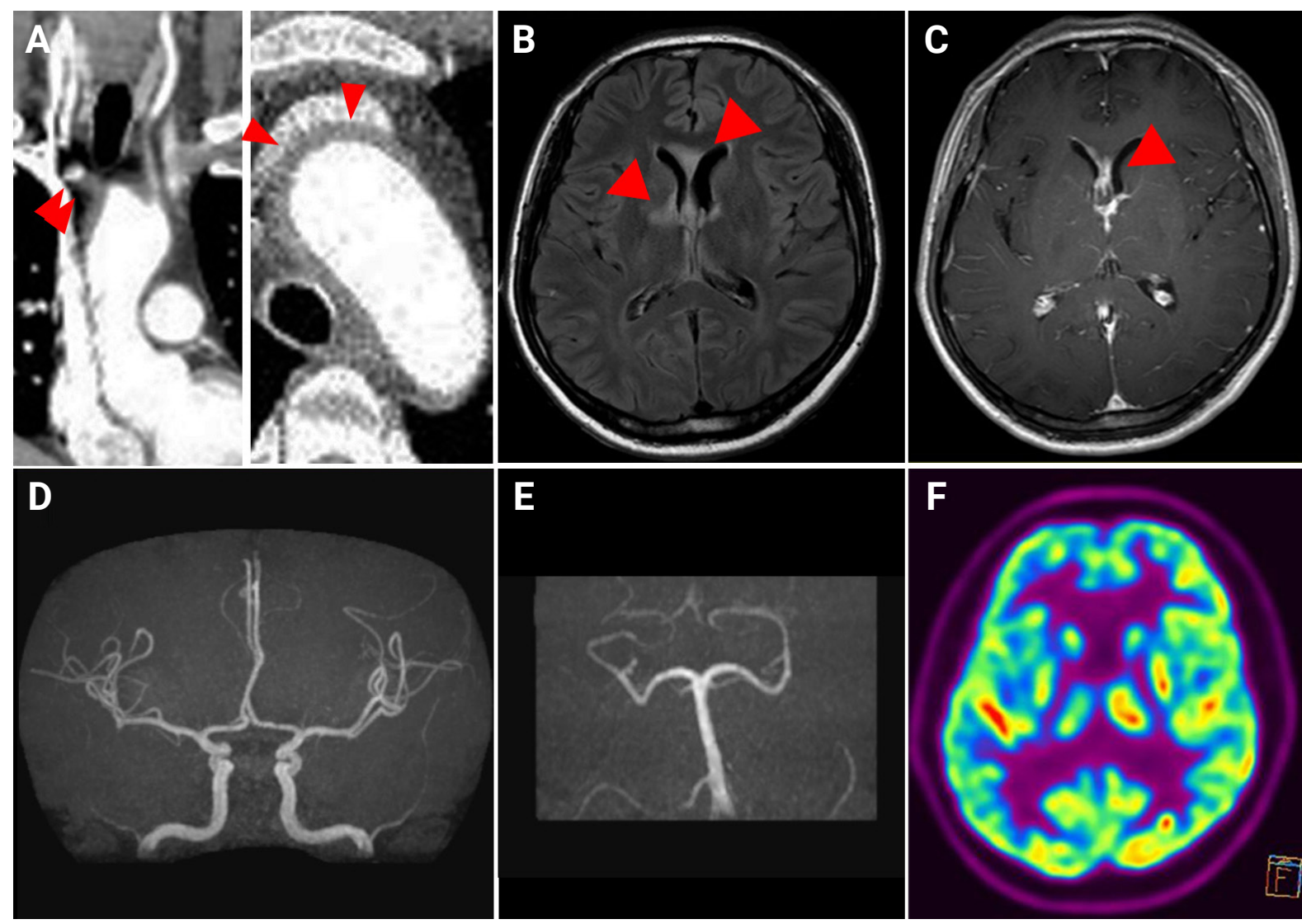

(A) Chest computed tomography shows thickening (arrowheads) at the ascending aorta and right proximal brachiocephalic artery. (B, C) Brain magnetic resonance imaging (MRI) shows the right basal ganglia and corpus callosum genu T2 fluid-attenuated inversion recovery high signal intensity with T1 enhancement (arrowheads). (D, E) Brain MRI shows the absence of intracranial steno-occlusive lesions. (F) Follow-up 18F-fluorodeoxyglucose positron emission tomography shows hypometabolism in the right basal ganglia. 
Figure 2 The disease course and treatment timeline

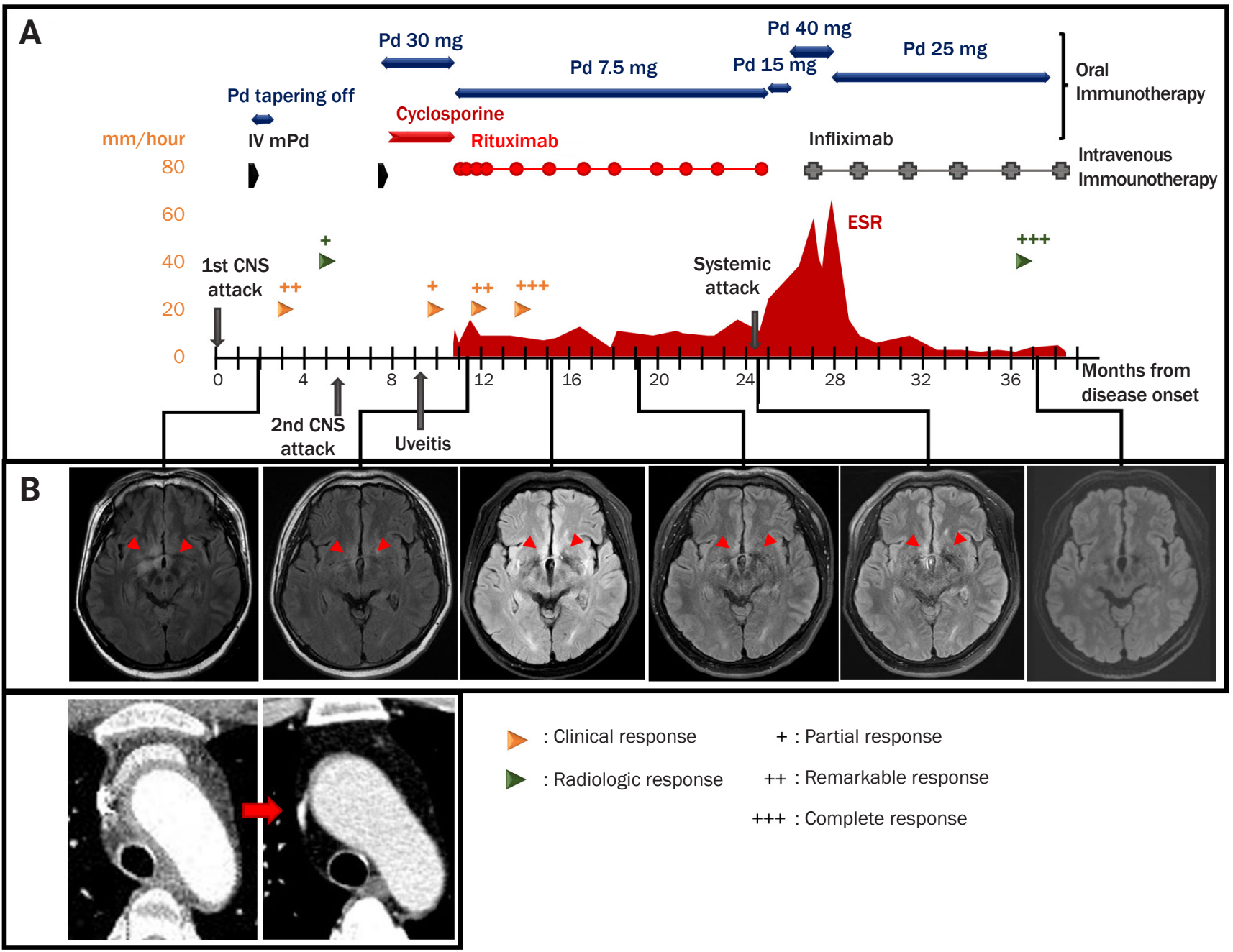

(A) The time of central nervous system (CNS) attack, uveitis, and systemic attack is indicated by the thick gray arrows. Colored triangles indicate clinical response (yellow) and radiologic response (dark green). The degree of response is represented by the number of ' + ' signs (one ' + ' sign $=$ partial response, two ' + ' signs $=$ remarkable response, and three ' + ' signs = complete response). Immunotherapy agents are represented by color shapes. The dark red graph demonstrates erythrocyte sedimentation rate (ESR) level ( $\mathrm{mm} /$ hour) started to increase with the systemic attack. (B) Brain magnetic resonance imaging shows both hypothalamic T2 fluid-attenuated inversion recovery high-intensity lesions. Thin lines connect brain image with the time of imaging. The lesions were persistent until infliximab was administered. (C) Follow-up chest computed tomography shows the lesion improving at the ascending aorta. Pd, oral prednisolone; IV mPd, intravenous methylprednisolone pulse therapy.

Approximately 4 months after the first steroid pulse therapy, the patient developed sudden memory impairment, recurrent headache, urinary incontinence, and sleeping tendency. Approximately 2 months after the recurrence, IV steroid pulse was readministered, followed by oral steroid and cyclosporine combination therapy. Despite immunotherapy, impaired cognitive function (subjectively, $80 \%$ of baseline status) and headache remained. Although the patient was treated with oral steroid and cyclosporine combination therapy, uveitis occurred approximately 6 weeks after the second steroid pulse therapy. Follow-up brain MRI (3 months after second pulse therapy) revealed a few residual patchy $\mathrm{T} 2$ high signal intensities in both the basal ganglia, hypothalamic areas, and optic tracts without enhancement and diffusion restriction.

Based on clinical symptoms, including remaining cognitive dysfunction, rituximab was administered as an alternative treatment. After five cycles of rituximab (375 mg/m²/cycle), the patient showed complete recovery of neurological symptoms. Although clinical response was remarkable, subsequent brain images showed no interval changes. 
Around the 12th cycle of rituximab, fever recurred with a painful erythematous patchy rash on the abdomen and right tibia. Erythrocyte sedimentation rate (ESR) was highly elevated. After infection was excluded, infliximab $(5 \mathrm{mg} / \mathrm{kg} /$ cycle every 2 months) was administered and ESR levels started to normalize (Figure 2A). Furthermore, there were no definite residual patchy $\mathrm{T} 2$ high signal intensities in the basal ganglia, hypothalamic area, or optic tracts, which was a complete radiological response after the five cycles of infliximab (Figure 2B). Around the eighth cycle of infliximab, wall thickening at the ascending aorta and its main branches were not observed on follow-up chest CT (Figure 2C).

\section{Discussion}

TAK is a large vessel vasculitis affecting the aorta and its major branches. However, TAK can exhibit extravascular involvement, including arthritis, erythema nodosum, and uveitis [8]. In the present case, uveitis occurred after the second CNS attack, and infliximab ameliorated erythema nodosum and CNS lesions. TAK and encephalitis could have occurred independently (i.e., coincidentally). However, the temporal association and complete responses of both systemic inflammation and CNS lesions to treatment indicated that encephalitis was an extravascular manifestation of TAK.

Notably, the persistent CNS lesions were reversed with infliximab. The persistent lesions indicated the remaining vasogenic edema was due to the ongoing inflammatory process. Although B cell immunity was suppressed by rituximab therapy and endothelial injury was restored, it was insufficient to reconstruct the disruption of the blood-brain barrier caused by other immune mechanisms. In contrast, infliximab therapy completely suppressed inflammation and induced a complete radiologic response, eliminating vasogenic edema.

Regarding immunological aspects, Mutoh et al. [9] recently reported antiendothelial cell antibodies promote vascular inflammation by activating endothelial cells in TAK. Our patient's clinical response of CNS symptoms to rituximab therapy supports this hypothesis. Despite the clinical response, the effect of rituximab on the patient's disease activity and radiological findings was insufficient. This result is compatible with the previous studies in which rituximab was suggested to have a role as second- or third-line but not first-line therapy in TAK [6]. Hypothetically, the B cell immunity does not play a critical role but an accessory role. In several previous studies, natural killer (NK) cells and CD8 T cells played essential roles [1]. T cells, NK cells, and macrophages produce TNF- $\alpha$ which is important in the formation of granulomatosis in TAK. Furthermore, TNF- $\alpha$ induces the production of interleukin-12 from macrophages, the key cytokine involved in CD4 T cell differentiation into $\mathrm{T}$ helper type 1 cells and NK cell activation. Infliximab is a TNF- $\alpha$ inhibitor and has been proven efficacious in TAK [7]. Although we anticipated infliximab would suppress the patient's disease activity and systemic manifestations, the complete resolution of CNS lesions was unexpected.

Notably, encephalitis associated with TAK was partially treatable with rituximab and completely with infliximab. To the best of our knowledge, this is the first report of the treatment options of TAK with direct CNS involvement.

In conclusion, rituximab and infliximab may be useful for managing the direct CNS involvement in TAK. Future investigations regarding the efficacy of this management are needed. However, this report is limited because CNS lesions could not be confirmed using brain biopsy.

\section{Conflicts of Interest}

Kon Chu has been on the editorial board of encephalitis since October 2020 and was not involved in the review process of the original article. The authors declare no other potential conflicts of interest relevant to this article.

\section{Author Contributions}

Conceptualization: Moon J, Park JK, Chu K; Data curation: Kim SD, Ahn SJ, Lee HS, Lee WJ; Investigation: Kim SD, Ahn SJ; Writing-original draft: Kim SD, Ahn SJ; Writing-review and editing: all authors.

\section{Acknowledgements}

There is no funding support for this manuscript.

\section{References}

1. Watanabe R, Berry GJ, Liang DH, Goronzy JJ, Weyand CM. Pathogenesis of giant cell arteritis and Takayasu arteritis-similarities and differences. Curr Rheumatol Rep 2020;22:68.

2. Zhang S, Yuan D, Tan G. Neurological involvement in primary systemic vasculitis. Front Neurol 2019;10:430

3. Bond KM, Nasr D, Lehman V, Lanzino G, Cloft HJ, Brinjikji W. Intracranial and extracranial neurovascular manifestations of Takayasu arteri- 
tis. AJNR Am J Neuroradiol 2017;38:766-772.

4. Chen YQ, Wang Y, Wang F, Wang NS. Proteus Takayasu's arteritis with unusual intracranial granulomatosis as initial manifestation. Rheumatol Int 2012;32:2185-2188.

5. Serra R, Butrico L, Fugetto F, et al. Updates in pathophysiology, diagnosis and management of Takayasu arteritis. Ann Vasc Surg 2016; 35:210-225.

6. Pazzola G, Muratore F, Pipitone N, et al. Rituximab therapy for Takayasu arteritis: a seven patients experience and a review of the literature. Rheumatology (Oxford) 2018;57:1151-1155.
7. Comarmond C, Plaisier E, Dahan K, et al. Anti TNF- $\alpha$ in refractory Takayasu's arteritis: cases series and review of the literature. Autoimmun Rev 2012;11:678-684.

8. Kwon OC, Lee SW, Park YB, et al. Extravascular manifestations of Takayasu arteritis: focusing on the features shared with spondyloarthritis. Arthritis Res Ther 2018;20:142.

9. Mutoh T, Shirai T, Ishii T, et al. Identification of two major autoantigens negatively regulating endothelial activation in Takayasu arteritis. Nat Commun 2020;11:1253. 